\title{
Gender Gap in Science
}

\section{A Global Approach to the Gender Gap in Mathematical, Computing, and Natural Sciences: How to Measure it, How to Reduce It?}

\section{by Mei-Hung Chiu and Mark Cesa}

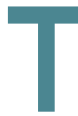

here continues to be a persistent gap between women's and men's participation, access, rights, pay, and benefits in the natural sciences, mathematics, and computing. The UNESCO Institute of Statistics reports that fewer than $30 \%$ of the world's researchers are women. Many scientists, mathematicians, computing experts, and policy makers are working to reduce this gender gap by way of a wide range of initiatives. The International Science Council (ISC) funded a unique three-year project in 2017-2019 called, "A Global Approach to the Gender Gap in Mathematical, Computing and Natural Sciences: How to measure it, how to reduce it?" that has provided a wide-ranging view of the issues women face in the sciences and how these issues may be overcome.

This project involves eleven partner organizations. Seven of these are union members of the ISC. The two lead organizations for the project are The International Mathematical Union (IMU), through its Committee for Women in Mathematics; and IUPAC (see IUPAC project 2017-007-1-020). Other participating unions include the International Union of Pure and Applied Physics (IUPAP); the International Astronomical Union (IAU), the International Union of Biological Sciences (IUBS); the International Council for Industrial and Applied Mathematics (ICIAM); and the International Union of History and Philosophy of Science and Technology (IUHPST). The other four organizations are the United Nations Educational, Scientific and Cultural

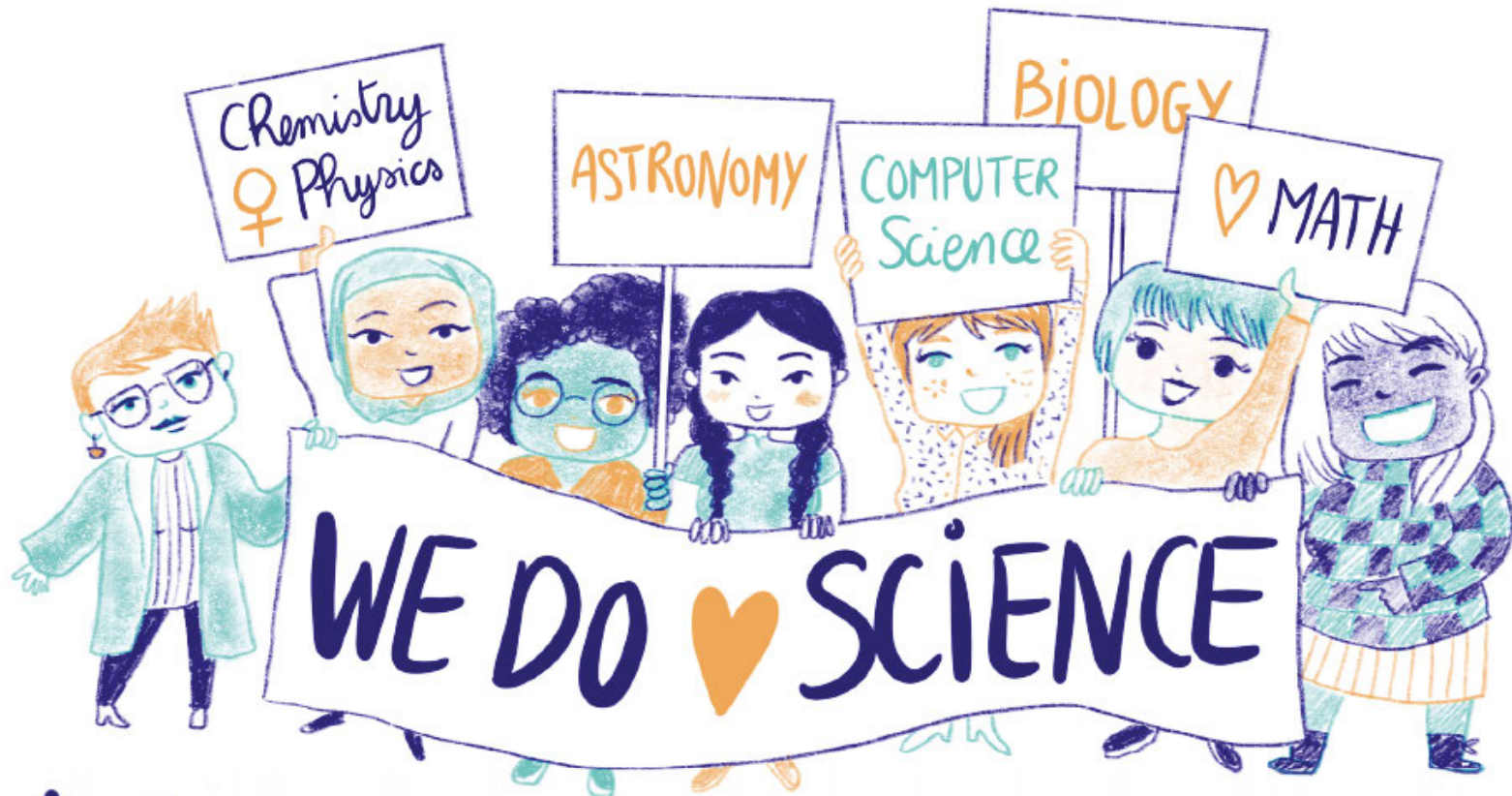

A Global Approach to the Gender Gap in Mathematical, Computing, and Natural Sciences: How to Measure It, How to Reduce 
Organization (UNESCO), through its project STEM and Gender Advancement (SAGA); Gender in Science, Innovation, Technology and Engineering (GenderlnSITE); the Organization of Women in Science for the Developing World (OWSD); and the Association for Computing Machinery (ACM), through ACM-W.

\section{Goals}

The main goal of the project was to investigate the gender gap in STEM disciplines through three principal tasks, globally and across disciplines. These main tasks were: (1) a global survey of scientists; (2) a study of scientific publication patterns; and (3) a database of good practices to encourage girls and young women to enter STEM fields. The primary focus of the project was on the academic profession, but information was gathered as well regarding conditions in industrial employment. The overall objective was to identify and promote tools, behaviors and practices to foster equality between men and women working in science by providing data from which to draw conclusions, take actions, implement policies to attract and retain women in STEM fields, and develop and evaluate practical recommendations.

\section{Findings}

\section{The Global Survey of Scientists}

The Global Survey of Scientists aimed to measure the gender gap in science around the world by investigating issues relating to the education and upbringing of young women; their career choices; workplace conditions and family obligations; and rates of participation and retention of women in the sciences, computing, and mathematics by comparing the experiences of women and men. The survey was conducted in seven languages (Arabic, Chinese, English, French, Japanese, Russian, and Spanish). To prepare the questionnaire, three regional meetings, in Colombia, South Africa, and Taiwan, were held to review the draft questionnaire with a special focus on: collecting feedback on regional implications of topics, providing unambiguous wording, gathering input to ensure that the survey was appropriate for all regions and for disciplines, and developing an outline for the distribution plan.

The Global Survey of Scientists was launched on 1 May 2018 and closed on 31 December 2018. There were more than 32,000 respondents to the first question, and analyses were performed on nearly 25,000 responses, including from 2,698 chemists, that contained sufficient data. Responses came from 159 countries,

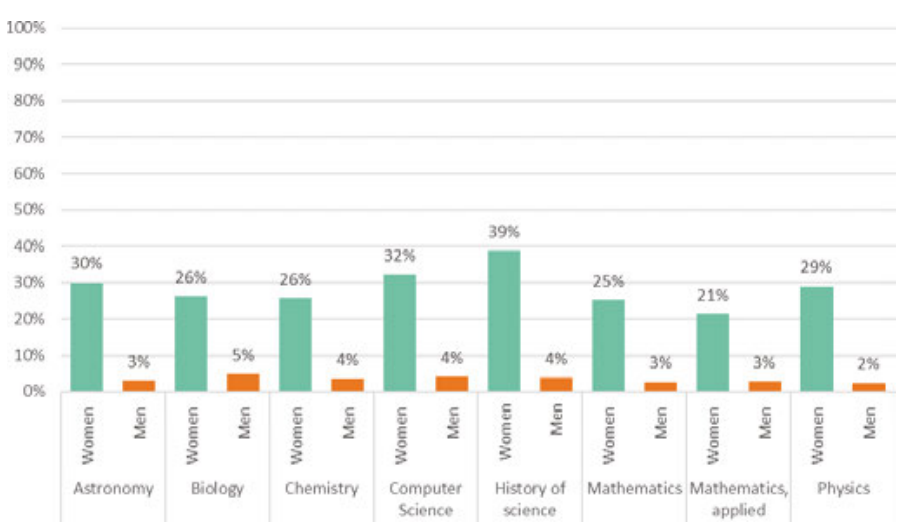

Figure 1. Respondents indicating they personally encountered sexual harassment at school or work, by discipline.

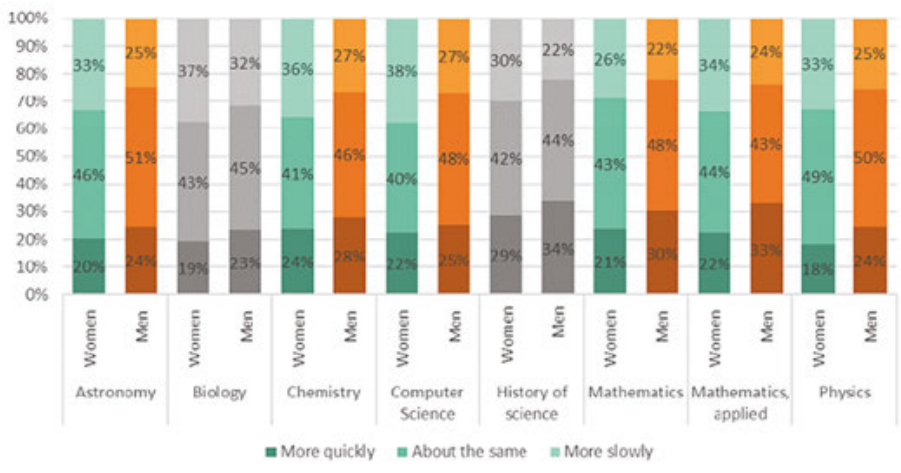

Grey indicates the differences are not statistically significant by gender on the 0.002 level.

Figure 2. Percentages of respondents comparing their career progression to their colleagues who completed their final degrees at the same time by discipline.

$100 \%$
$90 \%$
$80 \%$
$70 \%$
$60 \%$
$50 \%$
$40 \%$
$30 \%$
$20 \%$
$10 \%$
$0 \%$

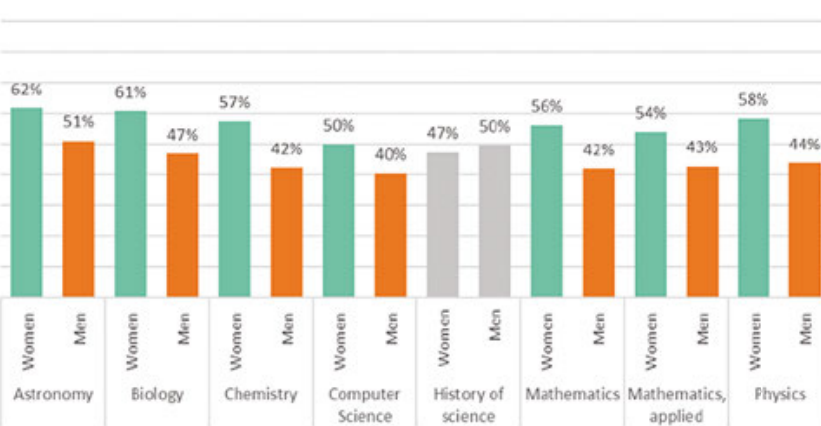

Each of these statistically significant relative differences ( $62 \%$ to $51 \%$, or 1.2 times, in Astronomy, for example) understates the relative likelihood between women and men in the multivariate model which accounts for confounding factors including age, employment sector, geographic region, and level of development.

Grey indicates the differences are not statistically signifcant by gender at the 0.002 level.

Figure 3. Percentages of respondents indicating their career influenced their decisions about children, marriage, or a similar long-term partnership by discipline. 


\section{The Global Gender Gap in Science}

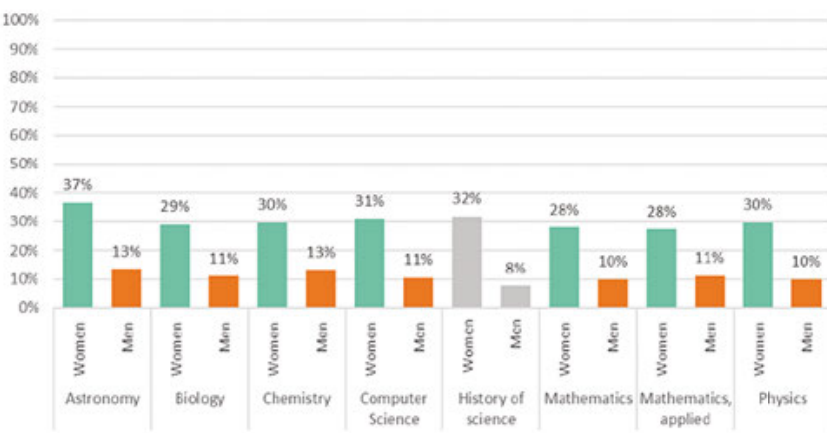

Each of these statistically significant relative differences (37\% to $13 \%$, or $~ 2.8$ times, in Astronomy, for example) understates the relative likelihood between women and men in the multivariate model which accounts for confounding factors including age, employment sector, geographic region, and level of development.

Grey indicates the differences are not statistically signifcant by gender at the 0.002 level.

Figure 4. Percentages of respondents indicating their career or rate of promotion slowed significantly because they becamne a parent by discipline.

and women and men are almost equally represented in the final dataset. Data were analyzed using three basic statistical approaches: bivariate, multivariate, and qualitative analyses. Data were collected using "snowball sampling," a technique in which existing respondents recruit others from among their colleagues, and partners in the project encouraged respondents in the partnering organizations, professional societies, and other relevant groups. Snowball sampling does not provide a statistically representative set of results, but they indicate trends among the respondents that were generally consistent across disciplines, geographical regions, and level of development.

In the final report of the Gender Gap project, results of the Global Survey of Scientists were reported in a graphical form that illuminated the differences between women's and men's experiences across disciplines, geographical regions, level of regional economic development, education, and employment sector.

It was found that systematic differences between the experiences of men and women still exist, across all regions, all disciplines, and all development levels; chemistry was not an exception.

The final report contains a great deal of information across the sciences. For chemistry in particular, some examples of the major findings are discussed here.

More than six times more women than men in chemistry reported having personally encountered harassment at work or school (Figure 1). This trend was essentially the same across disciplines, geographic regions, and levels of economic development.

Compared with men, women in chemistry tended to report more frequently that the progression of their careers was significantly slower than that of their peers (Figure 2). Women in chemistry also tended to report in greater numbers than men that their careers were more strongly affected by their decisions regarding children, marriage or similar long-term partnership, and that their careers were slowed significantly when they became parents (Figures 3 and 4)

There were also significant findings that spanned disciplines, regions and levels of economic development. For example, women in the survey were less likely than men to report respectful treatment by coworkers. Also, women were more likely than men to report that they took interruptions in their studies, and that the interruption had a negative impact on their professional credibility. More female respondents than male respondents expressed that they felt discriminated against

\begin{tabular}{|c|c|}
\hline Item & Gender Differences \\
\hline Doctoral advisor support ${ }^{+}$ & $\begin{array}{l}\text { Respondents from Math programs were more likely to rate advisor support higher than } \\
\text { respondents from other disciplines. }\end{array}$ \\
\hline Respectful co-workers ${ }^{+}$ & $\begin{array}{l}\text { Respondents whose discipline was Physics were more likely to agree they had respectful } \\
\text { co-workers than respondents from other disciplines. }\end{array}$ \\
\hline Never experienced discrimination ${ }^{+}$ & $\begin{array}{l}\text { Respondents from Math and and Physics were more likely to indicate they had never } \\
\text { experienced discrimination than respondents from other disciplines. }\end{array}$ \\
\hline Promotion slowed after children ${ }^{+}$ & $\begin{array}{l}\text { Respondents from Math were less likely to indicate their promotion slowed after becoming a } \\
\text { parent than respondents from other disciplines. }\end{array}$ \\
\hline No career change after children ${ }^{+}$ & $\begin{array}{l}\text { Respondents from Chemistry and Math were more likely to indicate their work or careeer did } \\
\text { not change when they became a parent than respondents from other disciplines. }\end{array}$ \\
\hline
\end{tabular}

These gender differences are statistically significant at the $\alpha=0.002$ level after accounting for potential confounding factors (age, discipline, geographic region, and level of development.)

tThe dependent variable is ordinal, so we are unable to report a single odds ratio.

Table 1. Differences across disciplines. 


\section{The Global Gender Gap in Science}
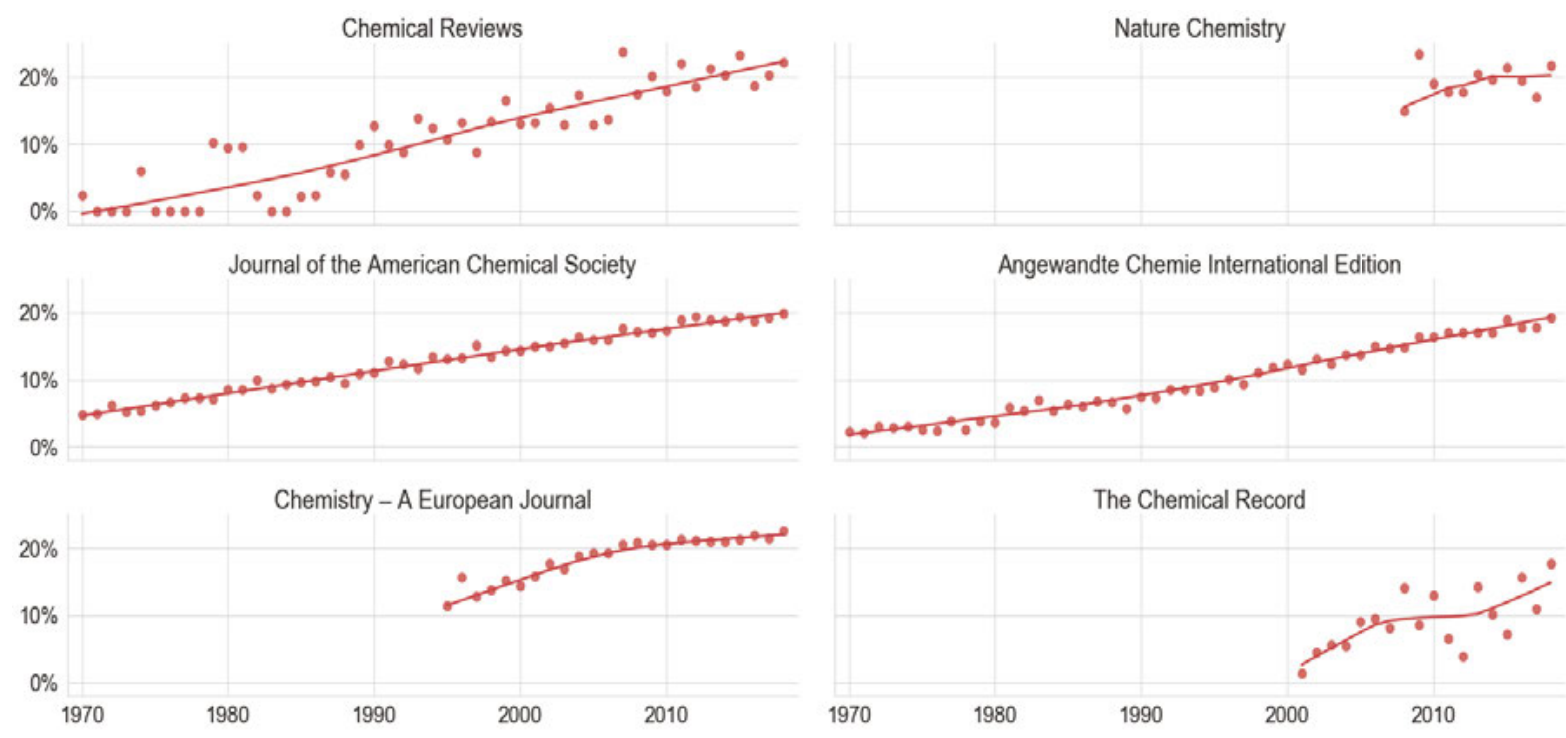

Figure 5. Trends in authorship by women in six major chemistry journals.

in the assessment or evaluation of their achievements because of their gender. Fewer women reported being treated fairly in their work environment, and women more frequently reported lower pay than that of their colleagues. Finally, the gender gap in science does not disappear with increasing levels of regional economic development, as defined by the Human Development Index, HDI. In some instances, a higher level of development was found to be correlated with more negative experiences by women in graduate program quality, and relationship with their graduate advisors.

Overall, these analyses provide compelling evidence that women and men do not have the same experiences in science, and that women's experiences are less positive than men's. There are gender differences in every area that was examined. In addition to findings from the survey regarding harassment and rates of career progression, the survey found: (See Table 1):

- Women are less likely than men to say that everyone is treated fairly in the educational system and in employment.

- Women report less positive relationships with their doctoral advisors, lower doctoral program quality, and more interruptions in doctoral studies than men.

- Women are less likely to report respectful treatment by co-workers than men.

\section{Study on Publication Patterns}

The analyses of publication patterns for women in mathematics, astrophysics, computer science and quantitative biology were based on data from three bibliographic sources managed by scientific organizations, namely: 1) The SAO/NASA Astrophysics Data System for research in astronomy and astrophysics, zbMATH for pure and applied mathematics; and arXiv for physics and mathematics and computer science or quantitative biology. However, in chemistry, the researchers used data on the representation of women in a selection of chemistry publications. Data from Crossref was used to investigate the proportion of women in 6 prestigious chemistry journals that were suggested by IUPAC volunteers: Chemical Reviews, the Journal of the American Chemical Society, Chemistry-A European Journal, Nature Chemistry, Angewandte Chemie International Edition, and The Chemical Record. The proportions of women authorship in these journals has been increasing steadily over the past 20 - 50 years (below $5 \%$ in 1970 up to values in the vicinity of $20 \%$ in the 2010s), a pattern that is not always repeated in other scientific disciplines (Figure 5).

There are overall improvements in academic career development for women as measured by increases in numbers of publications authored by women, although on a different pace depending on discipline. In particular, theoretical fields and sub-fields show the slowest improvement in most of the metrics applied, which leads to interesting questions such as the role of collaboration for the achievement of equal opportunities. However, the proportion of women authoring papers in top journals has remained static in mathematics and theoretical physics, while it increased in astronomy and chemistry. A pattern of fewer women authors in theoretical disciplines and sub-disciplines was found 


\section{The Global Gender Gap in Science}

while a larger presence of women was found in applied fields and with multiple authors. The rise of women representation in chemistry over the past five decades is perhaps the most noteworthy among the analyzed STEM disciplines. Even though the promising results were found, due to data limitation across the globe, much remains unexplained about how women chemical scientists on a global level.

\section{Database of good practices}

Various methods have been employed to gather information about initiatives for inclusion in the database, including holding conferences and conducting online searches. Countries in specific regions have been targeted through searches using the country name and a combination of key words (STEM, science, mathematics, gender, female, women, girls, mentor, role model, and workshop).

Creating the database has presented three challenges. The first challenge concerns the longevity of initiatives that are projects with finite funding (e.g., Horizon 2020 funded). Unless these projects have produced resources that can be found and used in future, they will not be included in the database. The second challenge is the difficulty in finding evidence of the effectiveness and impact of initiatives. The database of good practice currently includes 67 gender initiatives from more than 44 countries in all geographical regions of the world. Twenty-one initiatives come from Western Europe, followed by North America with 8 initiatives and Asia-Pacific with 7 initiatives. Among them, 24 initiatives relate to STEM education.

Finally, the final version of the database is to be hosted on the IMU/CWM website, and project partners will be able to make a link from their websites to the database.

\section{Resources}

Website: https://gender-gap-in-science.org/

Report: a book illustrated by a cartoonist is available on line at https://doi.org/10.5281/zenodo.3697222

Survey: the questionnaire in several languages is available at https://statisticalresearchcenter.aip.org/ global18, for the list of all the questions in English, see https://reurl.cc/yyzOxE

Interactive tools: for publication patterns, see https://reurl.cc/5gY30M, for a preliminary version of the database, see https://reurl.cc/gvKrLR.

Good practice: a selection of initiatives for reducing the gender gap, see the Database at https://www. mathunion.org/cwm/gender-gap-in-science-database

Research papers: several research papers will be submitted by the teams in charge of the three tasks.

Dissemination material: a 8-page illustrated document withmainideasand recommendationscanbefound at https://gender-gap-in-science.org/promotionalmaterials/

Creation of a community: 7 ISC unions (IMU, IUPAC, IUPAP, ICIAM, IUHPST, IAU, IUBS) and 4 other organizations (UNESCO, ACM, OWSD and Gender in Site) collaborated successfully. The three regional workshops and the final ICTP conference involved 150 active scientists all over the world.

\section{Recommendations}

Based on the findings of the project, the following recommendations were discussed and approved at the final Gender Gap project conference at ICTP in Trieste, Italy, in late 2019:

\section{For instructors and parents}

- Avoid gender stereotyping and unconscious gender bias in interactions with female students and children.

- Avoid books and social media that reinforce the gender gap in science. Develop gender awareness in the classroom and encourage girls in their learning of STEM subjects.

- Encourage relevant single-sex activities to raise girls' self-confidence and possibilities for expressing themselves.

\section{For local organizations}

- Promote a respectful, collegial working atmosphere in your organization.

- Define best practices to prevent, report and address sexual harassment and discrimination in professional spaces.

- Address the impact of parenthood on the careers of women. Encourage provision of a research-only year after maternity leave or parental leave.

- Ensure transparency of statistics on salaries, course loadings, bonuses, hiring and promotion, observing progress or difficulties experienced by female academics. Encourage policies to help reduce gendered salary disparities.

- Welcome families and provide child friendly environments. Provide improved support systems for parents.

- Address gender equality in all institutional policies. Identify a person or a group in charge of 
gender equality inside the organization, looking at the gender balance in all kind of activities.

- In all outreach and educational programs, include the aim of reducing the gender gap.

\section{For scientific unions}

- Work collectively to change culture and norms to reduce the various aspects of the gender gap.

- Define and advertise best practices to prevent, report and address sexual harassment and discrimination in professional spaces.

- Recommend and disseminate in the scientific community proper accounting of child bearing/ caring responsibilities (18 months per child recommended) when evaluating candidates in hiring and promotion processes.

- Actively promote the visibility of female scientists, in particular at conferences.

- Encourage the diversification of scientific awards, actively encouraging the nomination of women.

- Encourage the presence of women in editorial boards in your discipline and publish reports on the proportion of papers published by women.

- Welcome families in scientific activities.

- Create a committee for women and/or gender equality, with an assigned budget line.

- Actively promote gender balance at every level of your organization, including its leadership, its committees and also institutional events.

- In all outreach and educational programs and products, raise the awareness about the gender gap and include specific actions/events that aim at reducing the gender gap.

$\bullet$

\section{Next Steps}

Plans are under way to continue the work of the Gender Gap project to complete work on the major project tasks in 2020. Work will continue on collecting additional resources for good practices, as well as making the raw data from the global survey available to unions and interested scientists in a manner that assures confidentiality. Several of the unions are contributing financially and through volunteer participation. Finally, members of the Gender Gap project will discuss with the ISC its work on "Gender-transformative Science."

\section{Note}

This report was produced based upon the final report and booklet of the project. For details, please refer to https://doi.org/10.5281/zenodo.3697222 and https:// gender-gap-in-science.org/promotional-materials. Figures and drawings were taken from the final report.

\section{Acknowledgement}

Thanks to the representatives from the 11 unions and other $\mathrm{Pl} /$ contributors to the tasks of the project, in particular, for the leadership from Marie-Françoise Roy. These people are Marie-Françoise Roy (IMU, lead 1), Mei-Hung Chiu (IUPAC, lead 2), Irvy (Igle) Gledhill (IUPAP), Francesca Primas (IAU), Nathalie Fomproix (IUBS), Jean Taylor (ICIAM), Catherine Jami (IUHPST), and Jodi Tims (ACM). Task 1: Rachel Ivie, Mark Cesa, Laura Merner, Silvina Ponce Dawson, Susan White, and John Tyler; Task 2: Helena Mihaljević and Lucía Santamaría; Task 3: Merrilyn Goos and Regina Kelly. Also, thanks to Lea Castor for the drawings in this article.

\section{References}

1. A global approach to the gender gap in mathematical, computing, and natural sciences: How to measure it, How to reduce it? https://gender-gap-in-science.org/

2. UNESCO (2016). The SAGA Science, Technology and Innovation Gender Objectives List (STI GOL), SAGA Working paper 1. Paris. Isbn:978-92-2-100-154-3. url: https://unesdoc.unesco.org/ark:/48223/pf0000245006

\section{Mei-Hung Chiu is a professor at National Taiwan Normal University and a member of the IUPAC Executive Committee. Mark Cesa was president of IUPAC from 2014-2015.}

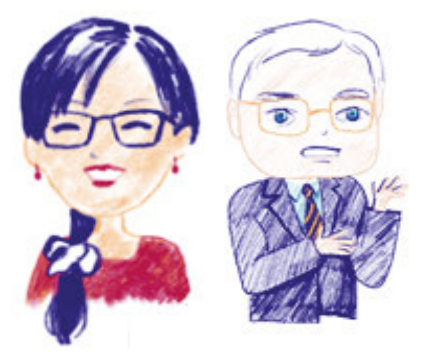

